\title{
Front Matter: Volume 11074
}

, "Front Matter: Volume 11074," Proc. SPIE 11074, Diffuse Optical Spectroscopy and Imaging VII, 1107401 (16 August 2019); doi: 10.1117/12.2542441

SPIE. Event: European Conferences on Biomedical Optics, 2019, Munich, Germany 


\title{
PROGRESS IN BIOMEDICAL OPTICS AND IMAGING
}

\section{Diffuse Optical Spectroscopy and Imaging VII}

\author{
Hamid Dehghani \\ Heidrun Wabnitz \\ Editors
}

\section{3-25 June 2019 \\ Munich, Germany}

Sponsored by

The Optical Society (United States)

SPIE

Published by

SPIE 
The papers in this volume were part of the technical conference cited on the cover and title page. Papers were selected and subject to review by the editors and conference program committee. Some conference presentations may not be available for publication. Additional papers and presentation recordings may be available online in the SPIE Digital Library at SPIEDigitalLibrary.org.

The papers reflect the work and thoughts of the authors and are published herein as submitted. The publisher is not responsible for the validity of the information or for any outcomes resulting from reliance thereon.

Please use the following format to cite material from these proceedings:

Author(s), "Title of Paper," in Diffuse Optical Spectroscopy and Imaging VII, edited by Hamid Dehghani, Heidrun Wabnitz, Proc. of SPIE-OSA Vol. 11074 (SPIE, Bellingham, WA, 2019) Seven-digit Article CID Number.

ISSN: 1605-7422

ISSN: 2410-9045 (electronic)

ISBN: 9781510628410

ISBN: 9781510628427 (electronic)

Copublished by

SPIE

P.O. Box 10, Bellingham, Washington 98227-0010 USA

Telephone +1 3606763290 (Pacific Time) · Fax +1 3606471445

SPIE.org

and

The Optical Society

2010 Massachusetts Ave., N.W., Washington, D.C., 20036 USA

Telephone 1 202/223-8130 (Eastern Time) · Fax 1 202/223-1096

http://www.osa.org

Copyright @ 2019, Society of Photo-Optical Instrumentation Engineers and The Optical Society

Copying of material in this book for internal or personal use, or for the internal or personal use of specific clients, beyond the fair use provisions granted by the U.S. Copyright Law is authorized by SPIE subject to payment of copying fees. The Transactional Reporting Service base fee for this volume is $\$ 21.00$ per article (or portion thereof), which should be paid directly to the Copyright Clearance Center (CCC), 222 Rosewood Drive, Danvers, MA 01923. Payment may also be made electronically through CCC Online at copyright.com. Other copying for republication, resale, advertising or promotion, or any form of systematic or multiple reproduction of any material in this book is prohibited except with permission in writing from the publisher. The CCC fee code is 1605$7422 / 19 / \$ 21.00$.

Printed in the United States of America by Curran Associates, Inc., under license from SPIE.

Publication of record for individual papers is online in the SPIE Digital Library.

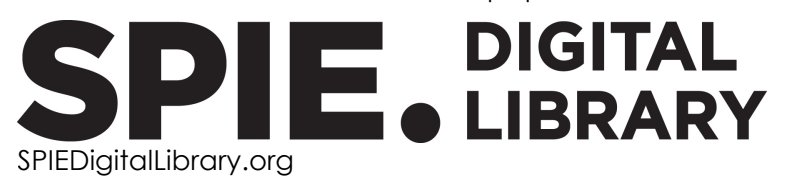

Paper Numbering: Proceedings of SPIE follow an e-First publication model. A unique citation identifier (CID) number is assigned to each article at the time of publication. Utilization of CIDs allows articles to be fully citable as soon as they are published online, and connects the same identifier to all online and print versions of the publication. SPIE uses a seven-digit CID article numbering system structured as follows:

- The first five digits correspond to the SPIE volume number.

- The last two digits indicate publication order within the volume using a Base 36 numbering system employing both numerals and letters. These two-number sets start with $00,01,02,03,04$, $05,06,07,08,09,0 A, 0 B \ldots$... OZ, followed by 10-1Z, 20-2Z, etc. The CID Number appears on each page of the manuscript. 


\title{
Contents
}

\author{
ix $\quad$ Authors \\ xiii Conference Committee \\ $\mathrm{xv}$ Introduction
}

\section{ADVANCES IN INSTRUMENTATION AND TECHNOLOGY I}

1107402 Large area SiPM and high throughput timing electronics: toward new generation time-domain instruments (Invited Paper) [11074-1]

$1107403 \quad$ Advances in wearable high-density diffuse optical tomography: first applications of a new commercial technology and development of an infant-specific research device [11074-2]

$1107404 \quad$ A wearable time domain near-infrared spectroscopy system [11074-3]

1107405 Integrating motion sensing and wearable, modular high-density diffuse optical tomography: preliminary results [11074-4]

$1107406 \quad$ A new multichannel broadband NIRS system for quantitative monitoring of brain hemodynamics and metabolism during seizures [1 1074-5]

\section{THEORY, ALGORITHMS AND COMPUTATIONAL TOOLS I}

$1107407 \quad$ Time-domain diffuse optical tomography with $I_{p}$ sparsity regularization for thyroid cancer imaging [1 1074-6]

1107408 Spectral approach to time domain diffuse optical tomography for breast cancer: validation on meat phantoms [1 1074-7]

1107409 Time-resolved diffuse optical tomography: a novel method to compute datatypes allows better absorption quantification [11074-8]

11074 OA Spatially-enhanced time-domain NIRS for determination of optical properties in layered structures [11074-9]

11074 OB Time-domain diffuse correlation spectroscopy quantifies path-length-resolved dynamical properties of a layered turbid media [11074-10]

11074 OC Simulation of fluorescence molecular tomography using a registered digital mouse atlas [11074-11] 
$11074 \mathrm{OE} \quad$ Mapping hemodynamic changes during hypoglycemia in the very preterm neonatal brain: preliminary results [11074-13]

$11074 \mathrm{OF} \quad$ Non-invasive optical assessment of intracranial pressure: pilot results in human patients [11074-14]

$11074 \mathrm{OH} \quad$ Cortical activity underlying overt and covert language generation measured using highdensity diffuse optical tomography [11074-16]

\section{COMPUTATION SOFTWARE AND ANALYSIS TOOLS}

$11074 \mathrm{OL} \quad$ Cloud-based NIRFAST server for tissue parameters recovery: laser and ultrasound co-analyser of thyroid nodules (Invited Paper) [1 1074-25]

11074 OM NeuroDOT: an extensible Matlab toolbox for streamlined optical functional mapping (Invited Paper) [11074-26]

THEORY, ALGORITHMS AND COMPUTATIONAL TOOLS II

11074 ON Visual appearance of blood vessels [1 1074-27]

11074 OP A broadband multi-distance approach to measure tissue oxygen saturation with continuous wave near-infrared spectroscopy [11074-29]

$110740 Q \quad$ Diffuse optical tomography with polarized light: a GPU-accelerated polarization-sensitive Monte Carlo simulations for efficient sensitivity kernel computation [1 1074-30]

11074 OR Modelling light propagation for fetal monitoring in utero [1 1074-31]

11074 OS Theoretical analysis of hemodynamic signal from the scalp in time-gated NIRS imaging using null source-detector separation [11074-32]

\section{CEREBRAL HEMODYNAMICS AND NEURAL ACTIVITY II}

11074 OT Time-resolved near infrared spectroscopy in ischemic stroke patients (Invited Paper) [1 1074-33]

$11074 \mathrm{OV}$ Short and mid-term reproducibility analysis of cerebral tissue saturation measured by time domain-NIRS [1 1074-35]

11074 0X Measurement of fetal cerebral blood flow of the lamb fetus in utero [11074-37] 
11074 OY New near-infrared spectroscopy method for local measurements of cerebral blood flow [11074-19]

$110740 Z$ Diffuse correlation spectroscopy for intracranial pressure estimation through cardiac pulse waveform analysis [11074-20]

1107410 The LUCA device: Iaser and ultrasound co-analyzer for thyroid nodules [11074-21]

$1107411 \quad$ In vivo time domain speckle contrast optical spectroscopy [11074-22]

1107412 Compressive sensing based hyperspectral bioluminescence imaging [11074-23]

1107413 A near-infrared hyperspectral imaging system for quantitative monitoring of hemodynamics and metabolism on the exposed cortex of mice [11074-24]

\section{NOVEL APPLICATIONS OF DIFFUSE OPTICS}

$1107414 \quad$ Multimodal measurements of brain tissue metabolism and perfusion in a neonatal model of hypoxic-ischaemic injury [11074-38]

1107415 Towards depth-resolved characterization of hemodynamics and oxygenation in the rat kidney [11074-39]

1107416 Diffuse reflectance bi-layer algorithm to enhance spoof detection of a TFT based biometry device [1 1074-40]

1107419 Functionalized upconversion luminescent nanoparticles for theranostics [1 1074-43]

\section{PHANTOMS AND PERFORMANCE ASSESSMENT}

11074 1A The BITMAP exercise: a multi-laboratory performance assessment campaign of diffuse optical instrumentation [1 1074-44]

11074 1B The BitMap dataset: an open dataset on performance assessment of diffuse optics instruments [11074-45]

11074 1C A solid phantom recipe for biophotonics applications: a step towards anatomically correct 3D tissue phantoms [11074-46]

$11074 \mathrm{lE} \quad$ Blood-lipid liquid phantom for assessing time and frequency domain tissue oximeter performances [1 1074-48] 


\section{CLINICAL APPLICATION OF DIFFUSE OPTICS}

11074 1G Deep variational autoencoders for breast cancer tissue modeling and synthesis in SFDI [11074-50]

1107411 Deep neural networks improve diagnostic accuracy of rheumatoid arthritis using diffuse optical tomography [11074-52]

$11074 \mathrm{lJ}$ The increase in amplitude of fluctuations of cerebral hemoglobin concentration in respiratory band related to increase in intra-abdominal pressure [1 1074-53]

11074 1K TD-fNIRS for diagnosing glaucoma: a clinical pilot study [1 1074-54]

$11074 \mathrm{lL} \quad$ Pilot measurement of the microvascular blood flow of thyroid nodules by diffuse optics [11074-55]

$110741 \mathrm{M}$ Diffuse reflectance spectroscopy for Onchocerca Volvulus nodules characterization [1 1074-56]

\section{POSTER SESSION}

1107410 Towards to deep neural network application with limited training data: synthesis of melanoma's diffuse reflectance spectral images [11074-66]

$11074 \mathrm{lP} \quad$ Monte-Carlo simulations of light transport in dense materials: dependent scattering and influence on sunscreen formulations [11074-58]

$110741 Q \quad$ Optical estimation of the composition of bone implants during processing [1 1074-59]

11074 IR Scanning and analysis of the surface of the chondroplasty zone in rabbits using Raman spectroscopy [11074-60]

11074 is Tissue fixation and substrate selection in hyperspectral imaging of murine models [11074-61]

11074 IT Modeling diffuse reflectance spectra of donated blood with their hematological parameters [11074-62]

11074 1V Non-invasive spectroscopic method of glucose concentration detemination in human blood: mathematical description [1 1074-64]

11074 IW Investigation of skin conditions producing similar reflectance spectra but different point spread functions in Monte Carlo simulation [1 1074-65]

$110741 \mathrm{X}$ Portable device to monitor skin condition with diffuse, multi-spectral illumination [1 1074-67]

$11074 \mathrm{IY}$ Efficient Monte Carlo simulations of spatially resolved reflectance for detection schemes with low numerical apertures [11074-68] 
$1107412 \quad$ Imaging of the optical properties of turbid media with integrated detection based on the Kubelka-Munk model [11074-69]

1107420 Experimental investigation on the light transmission of a textile-based over-cap used in functional near-infrared spectroscopy [11074-70]

1107421 Spatial-frequency-domain optical tomography in the radiative transport regime [11074-71]

1107422 Multi-wavelength time-resolved NIRS measurements for estimation of absolute concentration of chromophores: blood phantom study [11074-72]

1107423 Comparative spectral analysis of the component composition of bioimplants making in different ways for the treatment of gingival recession [1 1074-73]

1107424 Preliminary vastus lateralis characterization with time domain near infrared spectroscopy during incremental cycle exercise [11074-74]

1107425 Multimodal imaging platform for surgical guidance during epilepsy surgery [1 1074-76]

1107426 Fitting a spectral model for component analysis in diffuse optical tomography [11074-77]

1107427 Blood oxygenation in buried flaps: a bi-layer reconstruction [1 1074-78]

1107428 Experimental assessment of sensitivity profiles in multidistance time-resolved near infrared spectroscopy measurements [11074-79]

1107429 Robust calibration of reflectance acquired with optical fiber probes [1 1074-80]

$110742 \mathrm{~A}$ Direct determination of the spectrally resolved scattering phase function of suspensions and emulsions [11074-81]

11074 2C A new forward model for diffuse optical tomography [1 1074-83]

$110742 \mathrm{D}$ Spectral parameter recovery of cerebral and extra-cerebral tissues using broadband nearinfrared spectroscopy [11074-84]

$110742 \mathrm{E} \quad$ Reducing object curvature and height variation effects in hyperspectral images [1 1074-85]

$110742 \mathrm{G}$ Spectral determination of $\mu \mathrm{a}$, $\mu$ s and $\mathrm{g}$ of one thick turbid sample from three scattered light signals [1 1074-87]

$110742 \mathrm{H} \quad$ Frequency multiplexed intensity modulated diffuse reflectance system for quantification of tissue and arterial oxygen saturation [1 1074-88]

$1107421 \quad$ An adaptive scheme for diffuse-optical tomography based on combined structured-light illumination and single-pixel camera detection [11074-89]

$110742 \mathrm{~J} \quad$ Assessing extracerebral signal contamination in optical measurements of cerebral blood flow and oxygenation [1 1074-90] 
$110742 \mathrm{~K} \quad$ Pathlength distribution of (sub)diffusively reflected light [1 1074-91]

$110742 \mathrm{~L} \quad$ Influence of optical path length on multi-wavelength measurement of oxy- and deoxyhemoglobins in the skin [11074-93]

$110742 \mathrm{M} \quad$ Monitoring radiofrequency ablation of biological tissue using broadband time-resolved diffuse optical spectroscopy [1 1074-94]

$110742 \mathrm{~N} \quad$ Extended Kalman Filtering for the recovery of the absorption coefficients in layered turbid media [1 1074-95]

1107420 Construction of spectral reflectance database for estimation of absorption and scattering parameters in skin tissue [11074-96]

$110742 \mathrm{Q}$ Time-Resolved multi-wavelength, dual-channel system for diffuse optical spectroscopy: performance assessment [11074-98]

$110742 R \quad$ Instrument response function acquisition in reflectance geometry for time-resolved diffuse optical measurements [11074-99]

$110742 S \quad$ Diffuse correlation tomography in the transport regime: a theoretical study of the sensitivity to Brownian motion [1 1074-100]

$110742 \mathrm{~T} \quad$ Effects of ultrasound impedance matching fluids on diffuse optical measurements [1 1074-102]

$110742 \mathrm{U} \quad$ Compressive sensing time-domain Raman spectrometer for depth sensing of diffusive media [1 1074-103]

$110742 \mathrm{~V}$ In vivo time-domain diffuse correlation spectroscopy of the human muscle above $1000 \mathrm{~nm}$ [11074-104]

11074 2W Three-dimensional printed tissue-simulating phantoms for fluorescence imaging [1 1074-105] 


\section{Authors}

Numbers in the index correspond to the last two digits of the seven-digit citation identifier (CID) article numbering system used in Proceedings of SPIE. The first five digits reflect the volume number. Base 36 numbering is employed for the last two digits and indicates the order of articles within the volume. Numbers start with 00, 01, 02, 03, 04, 05, 06, 07, 08, 09, OA, OB...0Z, followed by 10-1Z, 20-2Z, etc.

Abdalmalak, Androu, 2J

Acharya, Deepshikha, $0 Z$

Aizu, Yoshihisa, IW, 20

Alves, Mariana, 06

Anazodo, Udunna, 14

Andersson-Engels, Stefan, OR, 1C

Aranda, Gloria, 10, 1L, 2T

Arao, K., 12

Arridge, Simon, 08, 26, 21

Azzarello, F., 24

Baez, Guido, 2N

Bainbridge, Alan, 14

Baker, Wesley B., OF

Bale, Gemma, 06, OP, 14

Balu, Ramani, OF

Baraldi, Eugenio, OE

Bassi, Andrea, 2

Beaudet, Jean, 16

Beaulieu, Emile, 25

Behera, Anurag, 02, 1A, 1B, 2Q

Bejm, Karolina, $1 \mathrm{~J}$

Belli, Antonio, 1A, 1B

Beltran-Gonzalez, A., IX

Belyaev, A. V., IV

Bentley, Alexander, 12

Berdún, Sergio, OX

Berger, Michel, 1M, 27

Betcke, Marta, 2 I

Bettega, Georges, 27

Blaney, G., OY

Bliznuks, Dmitrijs, 10

Bolochko, Katrina, 10

Bondarenko, Andrey, 10

Borycki, Dawid, OB

Bouthillier, Alain, 25

Bravo-Medina, B., $1 \mathrm{X}$

Bressel, Lena, IP

Brigadoi, Sabrina, 03, 05, OE

Burke, Ray, OR

Bürmen, Miran, 1Y, 29

Buttafava, Mauro, 04, 10, 2Q

Cantow, Kathleen, 15

Chizhov, Yuriy, 10

Clemente, P., 12

Climent, V., 12

Cobelli, Claudio, $0 \mathrm{E}$

Coll, Jean-Luc, 27

Colombo, L., 11, 2V

Colombo, R., $1 \mathrm{~K}$
Condat, Laurent, 09, 1B

Conde, Olga M., $1 G$

Contini, Davide, 02, 04, 0T, 10, 11, 1E, 1K, 1L, 24, $2 \mathrm{Q}, 2 \mathrm{R}, 2 \mathrm{~T}, 2 \mathrm{~V}$

Cooper, Robert J., 03, 05, 0E

Cortese, Lorenzo, OX, 10, 1L, 2T

Cross, J. Helen, 06

Cubeddu, Rinaldo, 0A, 1K, 2M

Culver, Joseph P., OH, OM, 11

Cutini, Simone, OE

Da Silva, Anabela, $0 Q, 2 S$

Dabrowski, Wojciech, $1 \mathrm{~J}$

Dalla Mora, Alberto, 02, 04, 08, 10, 11, 1A, 1B, 1L, 2Q, 2T, 2V

D'Andrea, Cosimo, 2I, 2U

Das, Kaustav, IW

de Fraguier, S., 10, $1 \mathrm{~L}$

De Roever, Isabel, 06

Dean, Emma, 06

Dehghani, Hamid, OL, OP, 10, 12, 1A, 1B, 11, 2D

Dell'Acqua, Roberto, $0 \mathrm{E}$

Di Sciacca, Giuseppe, 08, 26

Di Sieno, Laura, 02, 08, 11, 2Q, 2T

Diop, Mamadou, 14

Dolenec, Rok, is

Dolgyshkin, Dmitry A., IR

Dot, Audrey, 27

Duan, Jinming, $2 \mathrm{C}$

Dudnikov, S. Y., IV

Dunne, Luke, 03

Durduran, Turgut, OL, OX, 10, 11, 1A, 1B, 1L, 2S, 2T, 2V

Eggebrecht, Adam T., OH, OM

Eixarch, Elisenda, OX

Everdell, Nick, 03

Faber, Dirk J., 2K

Fantini, S., OY

Farina, Andrea, 08, 26, 2I, 2M, 2T, 2U

Feng, Yangqin, 11

Ferocino, Edoardo, 08, 26

Fishell, Andrew K., $\mathrm{OH}$

Flanders, Tracy M., OF

Flemming, Bert, 15

Flibotte, John J., OF

Forcione, Mario, 1A, 1B

Foschum, Florian, 2A

Francis, K. J., 2M

Frolov, O. O., 1Q, 23

Fujii, Hiroyuki, 07 
Funamizu, Hideki, 1W, 20

Galderisi, Alfonso, OE

Ganglani, Aman, 05

García, E., 10

García, Héctor, 2N

Garcia-Torales, G., $1 \mathrm{X}$

Gerega, Anna, 22

Germinario, Bruno, OT

Giacalone, Giacomo, OT

Giannoni, Luca, 13

Gjvaag, Terje, 20

Gladyłz, Thomas, 0A, 15

Gomis, R., 10, $1 \mathrm{~L}$

Goryacheva, Irina Yu., 19

Gratacós, Eduard, OX

Grosenick, Dirk, 0A, 15, 2N, 2W

Gunther, Jacqueline, OR

Gurevich, B. S., IV

Hanzu, F., 10, $1 \mathrm{~L}$

Hashisaka, Tomoki, 1W, 20

$\mathrm{He}$, Lian, OF

Hebden, Peter, 06

Henry, Maxime, 27

Hervé, Lionel, 09, 1A, 1B

Herzog, Bernd, IP

Hever, Gregory G., OF

Heye, Kristina, OF

Hill, Reuben, 03

Hirsch, Ole, 2W

Hoshi, Yoko, 07

$\mathrm{Hu}, \mathrm{Xin}-\mathrm{Hua}, 2 \mathrm{G}$

Isler, Helene, 1E, 22

Issar, Deepa, $0 Z$

Jallon, Pierre, 09, 16

Jauregui-Sánchez, Y., $1 Z$

Jayet, Baptiste, OR

Jezeršek, Matija, 2E

Jin, Jiahong, $2 \mathrm{G}$

Josè Torra, F., 10

Kacprzak, Michal, OB, $1 \mathrm{~J}$

Kainerstorfer, Jana M., $\mathrm{OZ}$

Kawaguchi, Hiroshi, 07

Kewin, Matthew, 14, 2J

Khalid, Mahro, 2J

Kienle, Alwin, ON, 2A

Koberling, Felix, 02

Kobori, Yuta, IW

Kochubey, Vyacheslav I., 19

Koenig, Anne, 16

Kofke, W. Andrew, OF

Konugolu Venkata Sekar, Sanathana, OA, 10, 11 ,

$1 \mathrm{C}, 1 \mathrm{~L}, 2 \mathrm{M}, 2 \mathrm{U}, 2 \mathrm{~V}$

Kovácsová, Zuzana, OP, 1A, 1B

Krämer, Benedikt, 02

Krischak, K., 10

Kruit, Hindrik, 2M

Lacerenza, M., 04, $2 \mathrm{U}$

Lakota, Katja, is

Lancis, J., $1 \mathrm{Z}$

Lange, Frédéric, OV, 13, 22
Lanka, Pranav, OA, 1A, 1B, 1C, 2M, 2Q

Lartizien, Rodolphe, 27

Laurence, Audrey, 25

Lazarev, Vladimir A., $1 R$

Leblond, Frederic, 25

Lenz, A. J. M., 1 Z

$\mathrm{Li}$, Haiyang, $1 \mathrm{C}$

Liang, Po-Jian, $2 \mathrm{H}$

Licht, Daniel J., OF

Liebert, Adam, OB, 1A, 1B, 1 J, 22, 28

Lighter, Daniel, 11

Lihachev, Alexey, 10

Lihacova, Ilze, 10

Likar, Boštjan, 1Y, 29

Liu, Linshan, 14

Liv, Yan, 0C

Lo Presti, Giuseppe, OX, 10, 1L, 2T

López-Higuera, José M., $1 \mathrm{G}$

LU, Jun Q., 2G

Lu, Wenqi, 2C

Macdonald, Callum M., 0Q, 2S

Macdonald, Rainer, OA

Machida, Manabu, 07, 21

Maeda, Takaaki, 20

Mahanna Gabrielli, Elizabeth, OF

Malek, Mokrane, 16

Maniewski, Roman, 1 J, 28

Manohar, Srirang, 2M

Marchesi, L., 04

Maria Frijia, Elisabetta, 03

Markel, Vadim A., OQ, 2S

Markova, Maria D., IR

Mars, Jérôme, 09, 1B

Martella, Pierluigi, 1C

Martelli, Fabrizio, 08

Marzorati, M., 24

Masuda, Yuji, 20

Mateo, Tony, 2T

Melih Can, Osman, $1 \mathrm{~T}$

Meseyarakov, V. D., 1Q

Messenio, D., $1 \mathrm{~K}$

Milanič, Matija, 1S, 2E

Milej, Daniel, 2J

Mimura, Tetsuya, 07

Mirtaheri, Peyman, 20

Mora, Mireia, 10, 1L, 2T

Morawiec, Magdalena, $1 \mathrm{~J}, 28$

Morrison, Laura, 14

Mutisya, Stephen M., 2 G

Naglič, Peter, 1Y, 29

Nakamura, G., OS

Navolokin, Nikita A., 19

Nguyen, Dang K., 25

$\mathrm{Ni}$, Ruiqing, $\mathrm{OC}$

Niendorf, Thoralf, 15

Nothelfer, Steffen, 2A

Ohya, Takehiro, IW

Okada, Eiji, 07, OS, 2L

Okawa, Shinpei, 07

Orive-Miguel, David, 09, 1A, 1B 
Ostojic, Daniel, 1E, 22

Oulhaj, Hind, $O Q$

Pacheco, Andrea, 1C

Pagliazzi, M., 11, 2V

Panizza, Pietro, 2T

Pardo, Arturo, $1 \mathrm{G}$

Pavlovčič, Urban, 2E

Pedrique, Belen, $1 \mathrm{M}$

Pernuš, Franjo, 1Y, 29

Perriollat, Mathieu, 27

Perše, Martina, 1s

Petitdidier, Nils, 16

Pham, T., OY

Pieropan, Edoardo, $\mathrm{OE}$

Pifferi, Antonio, 02, 04, 08, 0A, 10, 11, 1A, 1B, 1C,

$1 \mathrm{E}, 1 \mathrm{~L}, 26,2 \mathrm{M}, 2 \mathrm{~T}, 2 \mathrm{U}, 2 \mathrm{~V}$

Pinti, Paola, 06

Pirovano, lleana, 1K, 24, 2R

Planat-Chrétien, Anne, 1M, 27

Pogue, Brian W., $1 G$

Pohlmann, Andreas, 15

Polatoglu, Mavi Nunn, OC

Porcelli, S., 24

Post, Anouk L., 2K

Pouet, Marina, 16

Powell, Samuel, 03

Pugachov, E. I., 1Q

Pulawski, Przemyslaw, 1J

Rajaram, Ajay, 14, 2J

Re, Rebecca, OT, 1K, 24, 2R

Reich, Oliver, IP

Reighley, S., $1 \mathrm{X}$

Ren, Wuwei, 0C

Renna, Marco, 04, 10, 2Q

Ripoll, Jorge, OC

Rogelj, Luka, 2E

Rohilla, Sumeet, 02

Rosinski, Bogdan, 10, 1L, 2T

Roveri, Luisa, OT

Rowe, Jonathan E., 12

Rudin, Markus, OC

Ruesch, Alexander, $\mathrm{OZ}$

Samaei, Saeed, OB, 1A, 1B

Sassaroli, A., OY

Sawosz, Piotr, 0B, 1A, 1B, 1J, 22, 28

Schädel-Ebner, Sandra, $2 \mathrm{~W}$

Schira, Katrin, 2W

Schmitt, Samantha, $\mathrm{OZ}$

Schroeder, Mariel L., $\mathrm{OH}$

Seeliger, Erdmann, 15

Shahid, Marwan, 2J

Shapovalov, V. V., IV

Sherafati, Arefeh, $\mathrm{OH}$

Sherkat, Habib, 20

Shirai, T., 2L

Simončič, Urban, 2E

Smith, Greg, 03

Smith, Matthew A., OZ

Sparacino, Giovanni, OE

Spinelli, Lorenzo, OT, 1E, 1K, 24, 2R, 2V
Squarcia, Mattia, 10, 1L, 2T

St. Lawrence, Keith, 14, 2J

Stergar, Jošt, $1 S$

Strojnik, M., IX

Styles, lain B., 2C

Sudakou, Aleh, 1A, 1B, 22

Svoboda, Alexa M., $\mathrm{OH}$

Tachtsidis, llias, 06, OP, OV, 13, 14, 1A, 1B, 22

Tagliabue, Susanna, 1A, 1B

Tajahuerce, E., $1 Z$

Tanikawa, Yukari, 07

Taroni, Paola, 08, 10, 1L, 26, 2T

Thiebot, Alain, 16

Thornton, Rachel, 06

Tian, Peng, 2G

Tihov, Ivan, 23

Timchenko, Elena V., 1Q, 1R, 23

Timchenko, Pavrel E., 1Q, 1R, 23

Tomšič, Matija, $1 \mathrm{~s}$

Torricelli, Alessandro, 02, 04, 0T, 11, 1B, 1E, 1K, 24,

$2 \mathrm{R}, 2 \mathrm{~V}$

Tosi, Alberto, 04, 10, 2Q

Trevisanuto, Daniele, $\mathrm{OE}$

Tricoli, Ugo, OQ, 2S

Trudel, Dominique, 25

Tseng, Sheng-Hao, $2 \mathrm{H}$

Tuchin, Valery $V_{\text {., }} 19$

Ülgen, Yekta, $1 T$

Uteshev, Dilshat, 10

Valentini, Gianluca, 2I, 2U

van Leeuwen, Ton G., $2 \mathrm{~K}$

Veesa, Joshua Deepak, OP, 1A, 1B, 2C, 2D

Venturini, Elena, 2T

Vezyroglou, Aikaterini, 06

Vidal-Rosas, E. E., 11

Vidyasheva, Irina $\vee ., 19$

Volova, Larisa T., 1Q, 1R, 23

Wabnitz, Heidrun, OA, 1A, 1B, 1E, 2N

Wang, Yan, 11

Wanji, Samuel, $1 \mathrm{M}$

Weigel, Udo M., OL, 10, 1E, 1L

Wojak, Julien, $O Q$

Wojtkiewicz, Stanislaw, OL, 10

Wolf, Martin, OC, 1E, 22

Worley, Alan, 06

Yagofarova, E. F., 23

Yang, Jason, $\mathrm{OZ}$

Yang, Lin, OA, 1A, 1B

Yanina, Irina $Y u, 19$

Yodh, Arjun G., OF

Yuasa, Tomonori, 1W, 20

Zagorsky, I. G., IV

Zanoletti, Marta, OT, 10, 2Q

Zappa, F., 04

Zelinskyi, Yevhen, $1 Y$

Zhang, Lei, 11

Zhao, Hubin, 03, 05

Zolda, P., 10

Zoller, Christian, ON 
Proc. of SPIE-OSA Vol. 11074 1107401-12

Downloaded From: https://www.spiedigitallibrary.org/conference-proceedings-of-spie on 26 Apr 2023
Terms of Use: https://www.spiedigitallibrary.org/terms-of-use Terms of Use: https://www.spiedigitallibrary.org/terms-of-use 


\title{
Conference Committee
}

\author{
General Chair
}

Brett E. Bouma, Wellman Center for Photomedicine (United States)

Paola Taroni, Politecnico di Milano (Italy)

Programme Chairs

Ronald Sroka, Laser-Forschungslabor (Germany)

I. Alex Vitkin, University of Toronto (Canada)

\section{Conference Chairs}

Hamid Dehghani, The University of Birmingham (United Kingdom)

Heidrun Wabnitz, Physikalisch-Technische Bundesanstalt (Germany)

\section{Conference Programme Committee}

Wesley B. Baker, University of Pennsylvania (United States)

Regine Choe, University of Rochester (United States)

Davide Contini, Politecnico di Milano (Italy)

Jean-Marc Dinten, MINATEC (France)

Turgut Durduran, ICFO - Institut de Ciències Fotòniques (Spain)

Adam T. Eggebrecht, Washington University School of Medicine in St. Louis (United States)

Dirk Grosenick, Physikalisch-Technische Bundesanstalt (Germany)

Yoko Hoshi, Hamamatsu University School of Medicine (Japan)

Shudong Jiang, Thayer School of Engineering at Dartmouth (United States)

Jana M. Kainerstorfer, Carnegie Mellon University (United States)

Adam Liebert, Institute of Biocybernetics and Biomedical Engineering (Poland)

Eiji Okada, Keio University (Japan)

Felix Scholkmann, UniversitätsSpital Zürich (Switzerland)

Ilias Tachtsidis, University College London (United Kingdom)

Session Chairs

1 Advances in Instrumentation and Technology I

Davide Contini, Politecnico di Milano (Italy)

Wesley B. Baker, The Children's Hospital of Philadelphia

(United States) 
2 Theory, Algorithms and Computational Tools I

Hamid Dehghani, The University of Birmingham (United Kingdom)

3 Cerebral Hemodynamics and Neural Activity I

Yoko Hoshi, Hamamatsu University School of Medicine (Japan)

Adam T. Eggebrecht, Washington University School of Medicine in St. Lovis (United States)

4 Computation Software and Analysis Tools

Anabela Da Silva, Institut Fresnel (France)

5 Theory, Algorithms and Computational Tools II

Hamid Dehghani, The University of Birmingham (United Kingdom)

Ilias Tachtsidis, University College London (United Kingdom)

6 Cerebral Hemodynamics and Neural Activity II

Yoko Hoshi, Hamamatsu University School of Medicine (Japan)

Adam T. Eggebrecht, Washington University School of Medicine in St. Louis (United States)

7 Advances in Instrumentation and Technology II

Davide Contini, Politecnico di Milano (Italy)

Wesley B. Baker, The Children's Hospital of Philadelphia

(United States)

8 Novel Applications of Diffuse Optics

Ilias Tachtsidis, University College London (United Kingdom)

Adam Liebert, Nalecz Institute of Biocybernetics and Biomedical Engineering PAS (Poland)

9 Phantoms and Performance Assessment

Heidrun Wabnitz, Physikalisch-Technische Bundesanstalt (Germany)

Hamid Dehghani, The University of Birmingham (United Kingdom)

10 Clinical Application of Diffuse Optics

Jana M. Kainerstorfer, Carnegie Mellon University (United States)

Dirk Grosenick, Physikalisch-Technische Bundesanstalt (Germany) 


\section{Introduction}

The 2019 European Conferences on Biomedical Optics were held in Munich from June $23^{\text {rd }}-27^{\text {th }}$. The Diffuse Optical Spectroscopy and Imaging conference has continued to grow in the last decade and attracted over 105 submissions in 2019. This resulted in over 55 oral and 50 poster presentations from a range of international research groups, showing the significance of this conference for scientific exchange in this field in Europe and worldwide.

We have seen an enormous amount of development in Diffuse Optical Spectroscopy and Imaging, ranging from system development and testing to data analysis and advanced clinical applications. A major development seen in systems and technologies presented at this conference has been towards miniaturisation and wearable systems, both for continuous-wave spectroscopic and time-domain instrumentation. This enables new applications in complex real-life environments, such as comfortable measurements on young children or measurements during exercise in adults.

As diffuse optics technology has developed and our understanding of signal origins has improved, the use of multi-modality systems has also increased. Exciting work was presented to demonstrate that the use of broadband spectroscopic data together with diffuse correlation spectroscopy allows unique measurements of metabolism from the human brain to be obtained. The combination of optical and other technologies, such as ultrasound, was also prominent at the conference tackling important clinical problems such as detecting and characterising thyroid cancer.

Human brain health has remained a significant topic of research within the conference and many excellent contributions seeking a better understanding of brain health and identifying disease and trauma were presented. A look at these proceedings will show that the use of diffuse optics, being either through measurement of haemodynamic changes, oxygenation, or blood flow within humans is an active area of research. Many groups demonstrated validation of these measurements with current gold-standard techniques.

As system development has advanced, the community is becoming more aware regarding performance assessment and validation of instrumentation and data analysis. For the first time, we had two dedicated sessions to Phantoms and Standardisation as well as Open-Source Software which highlighted the importance of sharing knowledge in both areas.

This also included impressive work highlighting the importance of not only making well-characterized phantoms for system validation, but also of further developing them as a relevant factor in international standardisation. 
We are very excited to share these proceedings with the community and thank all contributors. We are confident that as the community grows, the importance and applications of diffuse optical imaging and spectroscopy will also continue to grow. We are looking forward to our next meeting in 2021.

Hamid Dehghani Heidrun Wabnitz 